\title{
Antenatal Diagnosis of Uterine Incarceration: Surgical Role of MRI and Intraoperative Transmyometrium Ultrasound
}

\author{
Masahito Mizuuchi',2,3*, Sakura Takada1, Masahiro Suzuki' ${ }^{1}$, Shin-ichi Ishioka², \\ Toshiaki Endo'2, Tsuyoshi Saito ${ }^{2}$ \\ ${ }^{1}$ Department of Obstetrics and Gynecology, Nikko Memorial Hospital, Muroran, Japan \\ ${ }^{2}$ Department of Obstetrics and Gynecology, Sapporo Medical University, Sapporo, Japan \\ ${ }^{3}$ Department of Obstetrics and Gynecology, Hakodate Municipal Hospital, Hakodate, Japan \\ Email: ${ }^{*}$ mizumasa@sapmed.ac.jp
}

Received 19 October 2014; revised 15 November 2014; accepted 8 December 2014

Academic Editor: Tarek Shokeir, Mansoura University Hospital, Egypt

Copyright (C) 2014 by authors and Scientific Research Publishing Inc.

This work is licensed under the Creative Commons Attribution International License (CC BY).

http://creativecommons.org/licenses/by/4.0/

(c) (i) Open Access

\section{Abstract}

Uterine incarceration is a rare condition thought to result from incarceration of a retroverted uterus in the small pelvis. The present case report describes a case of uterine incarceration caused by extensive pelvic adhesions after appendectomy for perforated appendicitis. A 39-year-old primigravida woman was referred to our obstetric unit for follow-up after successful in vitro fertilization. The symptoms related to uterine incarceration included anuria at 15 weeks of gestation and uterine contractions starting at 28 weeks of gestation. The absence of a cervical canal on ultrasonography is a key finding required to diagnose this rare condition. In the present case, the incision for uterotomy was determined by prenatal magnetic resonance imaging and transmyometrium ultrasonography during cesarean section. Both MRI and ultrasound images clearly showed anatomical relationships between the bladder, cervical canal and uterine wall. Clinicians need to know how the incision for uterotomy should be made and to avoid serious complications during cesarean section.

\section{Keywords}

Uterine Incarceration, Antenatal Diagnosis, Magnetic Resonance Imaging, Transmyometrium Ultrasonography

\footnotetext{
${ }^{*}$ Corresponding author.
}

How to cite this paper: Mizuuchi, M., et al. (2014) Antenatal Diagnosis of Uterine Incarceration: Surgical Role of MRI and Intraoperative Transmyometrium Ultrasound. Open Journal of Obstetrics and Gynecology, 4, 1082-1086. 


\section{Introduction}

Incarceration of the retroverted gravid uterus is a rare condition during pregnancy, and is diagnosed in 1 in 3000 pregnancies [1]. The risk factors for uterine incarceration include previous pelvic surgery, pelvic inflammatory disease, endometriosis, or leiomyoma [2] [3]. The prenatal diagnosis of uterine incarceration is important for determining the mode of delivery_cesarean section is often required, as vaginal delivery is impossible in most cases. Moreover, ultrasonography before and during cesarean section and prenatal magnetic resonance imaging (MRI) are useful for surgical management. This case report describes a case of uterine incarceration resulting from peritonitis after appendectomy for perforated appendicitis and importance of antenatal MRI and intraoperative transmyometrium ultrasound to determine the incision for uterotomy.

\section{Case Presentation}

A 39-year-old primigravida woman was referred to our tertiary obstetrics unit for follow-up at 12 weeks of gestation. Her surgical history included an open appendectomy for pan-peritonitis secondary to perforated appendicitis, performed 17 years earlier. This pregnancy was achieved by in vitro fertilization (IVF) after a 5-year history of primary infertility. At 15 weeks of gestation, the pregnant woman was referred to our emergency unit with anuria. Following the insertion of a Foley catheter for urinary retention, $500 \mathrm{~mL}$ of urine was drained. After this procedure, she was able to void without catherization and no further examination was performed.

At 20 weeks of gestation, she underwent routine screening transvaginal ultrasonography for preterm labor; the cervical canal could not be visualized and a diagnosis of total placenta previa was suspected (Figure 1). No vaginal bleeding or uterine contractions were observed. The examination was repeated after 4 weeks, and the findings were unchanged. During the speculum examination, the vaginal portion of the cervix could not be seen, the posterior vaginal fornix was swollen, and the cervix was not palpable. At this time, uterine incarceration was suspected, and a pelvic MRI examination was performed at 31 weeks' gestation (Figure 2). The MRI examination revealed that the cervix of the uterus was elongated and the fundus of the uterus was located in the pouch of Douglas. The placenta was located on the posterior wall of the uterus and there were sufficient margins between the edge of the placenta and the internal uterine os. At 31 weeks of gestation, a diagnosis of uterine sacculation was made on the basis of the MRI and speculum examination findings.

After 28 weeks of gestation, the number of uterine contractions increased and oral ritodrine hydrochloride was administered as a tocolytic agent. It was not so urgent condition of threatened premature labor, we did notuse antental corticosteroids therapy to enhance lung maturity. After 34 weeks of gestation, the patient was hos-

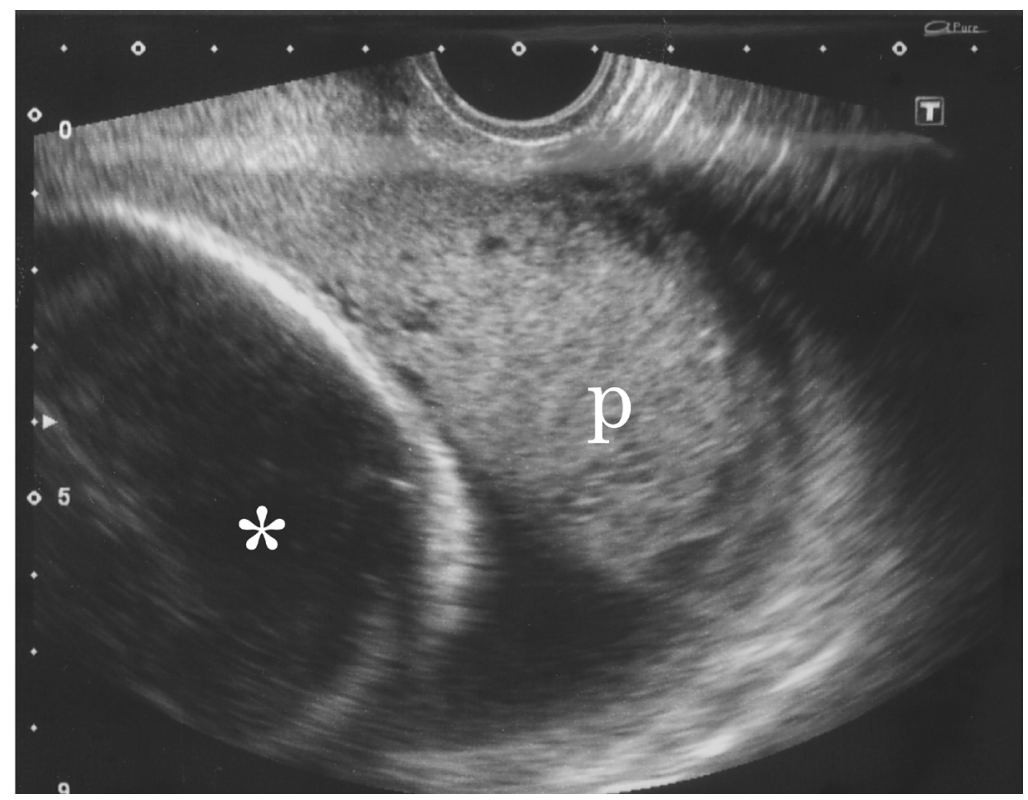

Figure 1. Transvaginal ultrasound view at 20 weeks' gestation mimicking placenta previa. The cervical canal cannot be visualized. p, placenta; ${ }^{*}$ Fetal head. 


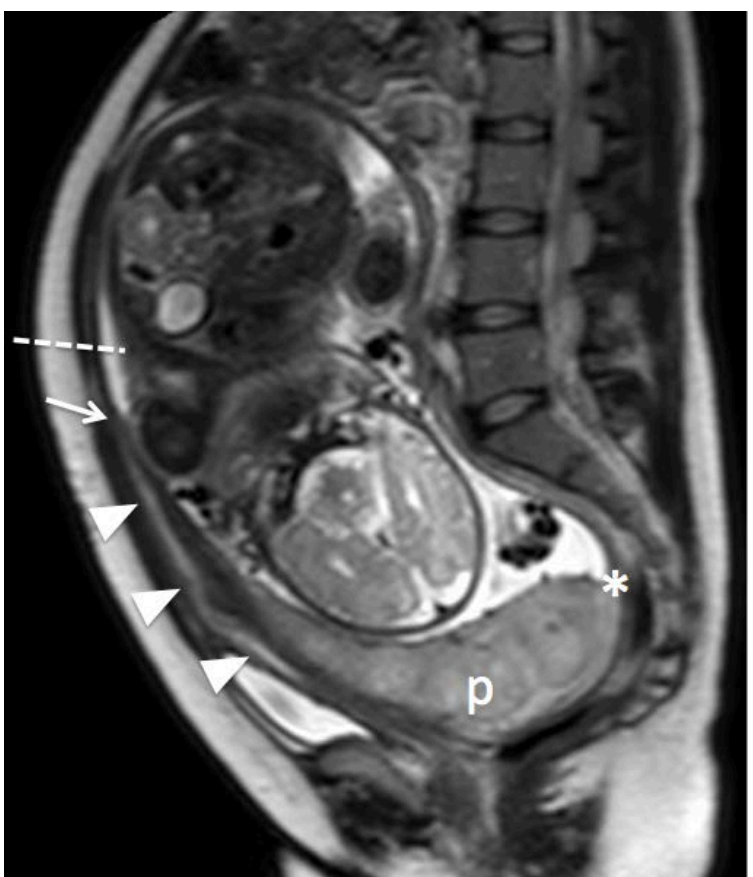

(a)

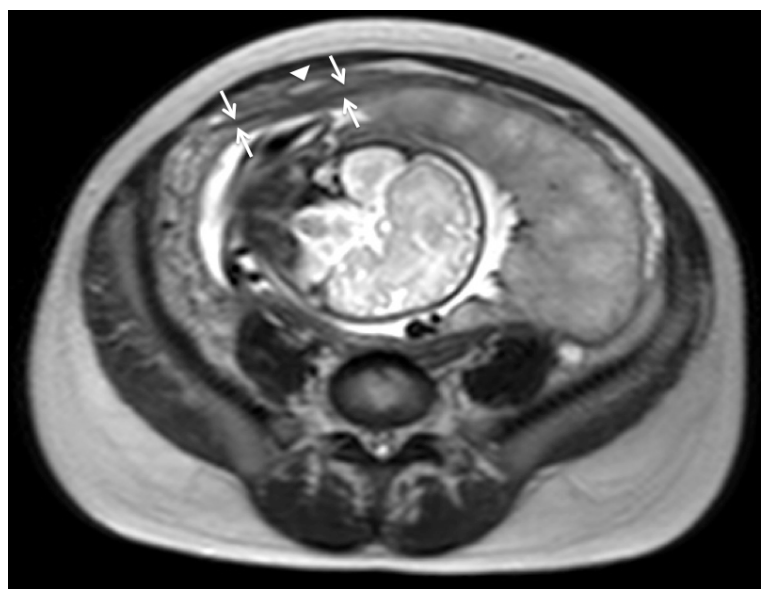

(b)

Figure 2. Magnetic resonance images at 34 weeks' gestation (T2WI, [a, sagittal view; b, axial view]). (a) The cervix (arrowhead) is extremely elongated. The incarceration of the uterus $\left(^{*}\right)$, including the fetal head and placenta (p), in the pouch of Douglas. This sagittal view shows the placenta placed in the posterior wall of the uterus and has sufficient margins from the internal cervical os (arrow). The level of the uterotomy is indicated by the dotted line (...), located above the internal os to avoid cervix and posterior uterine wall injuries; (b) Low T2 signal intensity (arrows) behind the cervix showing the space between the cervix and posterior uterine wall in the axial view.

pitalized with threatened preterm labor and received ritodrine hydrochloride intravenously. Although magnesium sulfate was added at 35 weeks, a cesarean section had to be performed at 36 weeks 0/7 of gestation under regional anesthesia, due to tocolysis failure and uterine incarceration.

The abdomen was entered through a midline incision up to the left side of the umbilicus. There were abnormal intramembranous adhesions between the fundus and the posterior wall of the uterus and intrapelvic organs, especially the rectum. As the adhesions could not be completely removed, we attempted to remove the minimum number of adhesions required to safely perform the cesarean section. Before making the myometrium incision, we performed ultrasonography directly from the myometrium (Figure 3). On ultrasonography, the anatomical relation between the internal os and the elongated cervical canal could be clearly visualized, thus facilitating the determination of the incision line of the myometrium to avoid cervical and placental injuries. Uterotomy was performed by lower transverse incision of the uterus indicated (dotted line, Figure 2(a)).

Although the fetus seemed to be in the cephalic position, the baby was born in the breech position. A male infant weighing $2406 \mathrm{~g}$ was delivered, with Apgar scores of 8 and 9 at 1 and 5 minutes, respectively, and an umbilical artery blood $\mathrm{pH}$ of 7.31. The baby was referred to the neonatal care unit because of polypnea, and was subsequently diagnosed with transient tachypnea of the newborn.

The placenta was removed manually without any difficulties and oxytocin was injected in the myometrium of the uterus. Finally, we confirmed that the incision was located in the lower uterine segment, near the internal cervical os, and did not involve the cervix or vagina. The duration of the operation was 80 minutes and the total blood loss was $1040 \mathrm{~g}$; 2 units of autologous blood was required for transfusion, which was ordered before the operation. The mother and the infant were discharged 7 and 12 days after delivery, respectively.

\section{Discussion}

Uterine incarceration is a rare condition in which the uterus is incarcerated in the small pelvis during pregnancy, and occurs in 1 in 3000 pregnancies [1]. Uterine retroversion is seen in approximately 15\% of pregnancies and 


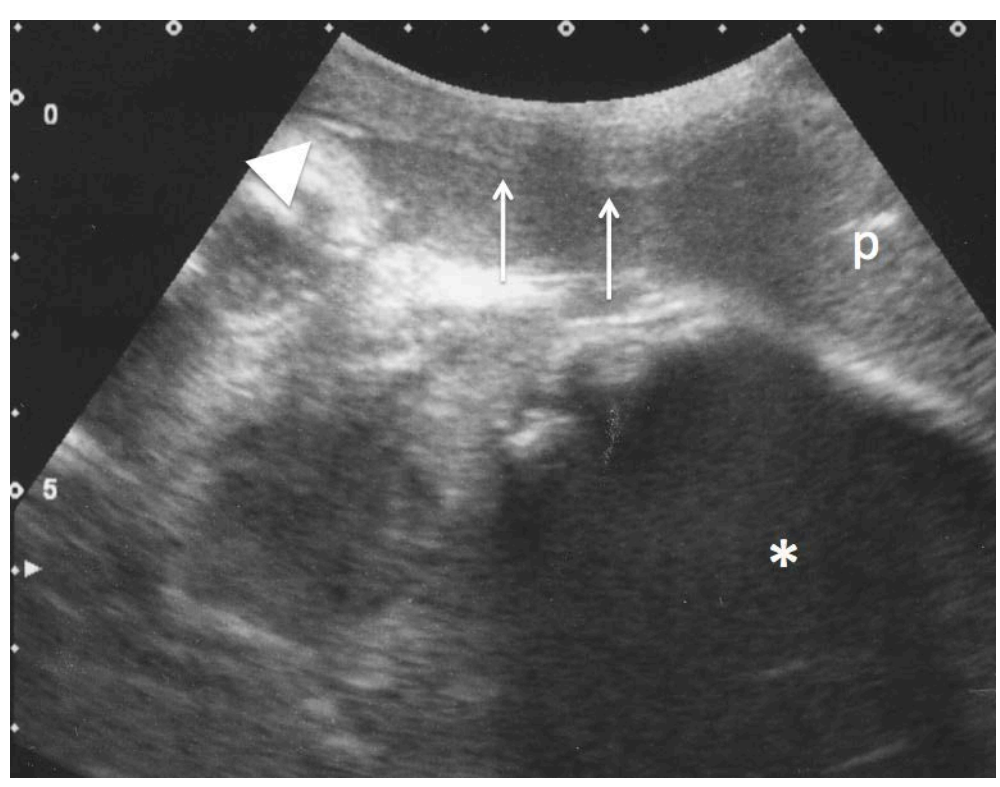

Figure 3. Intraoperative transmyometrium ultrasound image (sagittal view) at 36 weeks' gestation showing the relationships between the internal os (arrowhead) and placenta (p). Cervical canal (double arrows) is clearly shown and the fetal head $\left({ }^{*}\right)$ is visible. The image shows the cervical canal (double arrows) and internal os (arrowhead). $\left(^{*}\right)$ placenta; $\left(\left(^{* *}\right)\right.$ fetal head.

resolves spontaneously by 14 weeks of gestation, in most cases [4]. The most common symptoms associated with incarceration are lower abdominal pain, urinary retention, and constipation [5]. However, asymptomatic incarceration may also be present before labor. The risk factors of uterine incarceration include myoma uteri, uterine malformations, and intrapelvic adhesions attributable to endometriosis and previous surgery [2] [3]. In the present case, the appearance of the intra-abdominal area showed firm adherence of the fundus of the uterus and posterior pelvic wall; thus, we diagnosed the patient with uterine incarceration caused by intrapelvic adhesions. Other high risk factors such as myoma uteri, endometriosis, uterine malformations, and pelvic abnormalities were not present.

Without proper diagnosis and treatment, asymptomatic incarceration can cause spontaneous miscarriage, intrauterine fetal death, uterine rupture, preterm delivery, postpartum hemorrhage, and maternal acute renal failure [6] [7]. In particular, prenatal diagnosis is important to determine the mode of delivery and prevent complications such as uterine rupture during labor or cervical and fetal injuries in cesarean section [8]. In this case, failure to diagnose uterine incarceration before the surgery would have led to a uterine incision being made in the normal position; thus, cutting of the cervix and the posterior wall of the uterus would have been unavoidable during delivery. So it is very important to determine the incision for uterotomy before cesarean section. The diagnosis of uterine incarceration is made on the basis of the findings from the pelvic examination, high-resolution transabdominal ultrasound of the uterus with full bladder, and clinical history. Moreover, an MRI examination is recommended when uterine incarceration is highly suspected [9].

However, this rare condition cannot be easily diagnosed in routine examinations. In the present case, the condition was suspected after we failed to visualize the internal uterine os on vaginal ultrasonography at 24 weeks of gestation. Although the internal uterine os was not visualized on transvaginal ultrasonography, it was easily detected on MRI examination. The MRI examination clearly showed an elongated cervical canal and internal os (Figure 2(a)). Thus, the MRI examination is useful for diagnosing uterine incarceration and essential for determining the incision line of the uterine myometrium in cesarean sections. And also intraoperative transmyometrium ultrasound is useful to confirm the incision line to avoid cutting the cervical canal or bladder during cesarean section (Figure 3). As described in this case, the absence of the cervical canal on transvaginal ultrasound examination might be one of the key findings suggesting uterine incarceration, especially in cases with a history of pelvic adhesions. With the increasing rate of IVF-embryo transfer (ET) pregnancies, the number of patients with intra-abdominal adhesions and uterine incarceration may increase. Thus, transvaginal ultransonography 
should be performed with a clear understanding of this rare condition.

In the first trimester, manual repositioning of the uterus has been reported as a treatment for uterine incarceration [10]; however, in cases with extensive intra-abdominal adhesions, the adhesions may be difficult to detach manually and might cause complications such as intra-abdominal hemorrhage or small bowel perforation. The cause of the uterine sacculation needs to be determined before attempting manual repositioning in uterine incarceration.

In summary, although uterine incarceration is a rare complication during pregnancy, obstetricians should consider this diagnosis in pregnant women with symptoms of anuria or a cervical canal that is difficult to visualize on ultrasonography. And this case report clearly shows that the importance of antenatal MRI and intraoperative transmyometrium ultrasound which contribute to determine the incision for uterotomy to avoid serious surgical complications during caesarian section for uterine incarceration.

\section{References}

[1] Gibbons Jr., J.M. and Paley, W.B. (1969) The Incarcerated Gravid Uterus. Obstetrics and Gynecology, 33, $842-845$.

[2] Sweigart, A.N. and Matteucci, M.J. (2008) Fever, Sacral Pain, and Pregnancy: An Incarcerated Uterus. Western Journal of Emergency Medicine, 9, 232-234.

[3] Jacobsson, B. and Wide-Swensson, D. (1999) Incarceration of the Retroverted Gravid Uterus-A Review. Acta Obstetricia et Gynecologica Scandinavica, 78, 665-668. http://dx.doi.org/10.1080/j.1600-0412.1999.780801.X

[4] Frei, K.A., Duwe, D.G., Bonel, H.M., Dürig, P. and Schneider, H. (2005) Posterior Sacculation of the Uterus in a Patient Presenting with Flank Pain at 29 Weeks of Gestation. Obstetrics and Gynecology, 105, 639-641. http://dx.doi.org/10.1097/01.AOG.0000141695.83741.94

[5] Lettieri, L., Rodis, J.F., McLean, D.A., Campbell, W.A. and Vintzileos, A.M. (1994) Incarceration of the Gravid Uterus. Obstetrical \& Gynecological Survey, 49, 642-646. http://dx.doi.org/10.1097/00006254-199409000-00026

[6] Weekes, A.R., Atlay, R.D., Brown, V.A., Jordan, E.C. and Murray, S.M. (1976) The Retroverted Gravid Uterus and Its Effect on the Outcome of Pregnancy. British Medical Journal, 1, 622-624. http://dx.doi.org/10.1136/bmj.1.6010.622

[7] Newell, S.D., Crofts, J.F. and Grant, S.R. (2014) The Incarcerated Gravid Uterus: Complications and Lessons Learned. Obstetrics and Gynecology, 123, 423-427. http://dx.doi.org/10.1097/AOG.0000000000000102

[8] De Friend, D.E., Dubbins, P.A. and Hughes, P.M. (2000) Sacculation of the Uterus and Placenta Accreta: MRI Appearances. The British Journal of Radiology, 73, 1323-1325. http://dx.doi.org/10.1259/bjr.73.876.11205679

[9] Fernandes, D.D., Sadow, C.A., Economy, K.E. and Benson, C.B. (2012) Sonographic and Magnetic Resonance Imaging Findings in Uterine Incarceration. Journal of Ultrasound in Medicine, 31, 645-650.

[10] Seubert, D.E., Puder, K.S., Goldmeier, P. and Gonik, B. (1999) Colonoscopic Release of the Incarcerated Gravid Uterus. Obstetrics and Gynecology, 94, 792-794. 
Scientific Research Publishing (SCIRP) is one of the largest Open Access journal publishers. It is currently publishing more than 200 open access, online, peer-reviewed journals covering a wide range of academic disciplines. SCIRP serves the worldwide academic communities and contributes to the progress and application of science with its publication.

Other selected journals from SCIRP are listed as below. Submit your manuscript to us via either submit@scirp.org or Online Submission Portal.
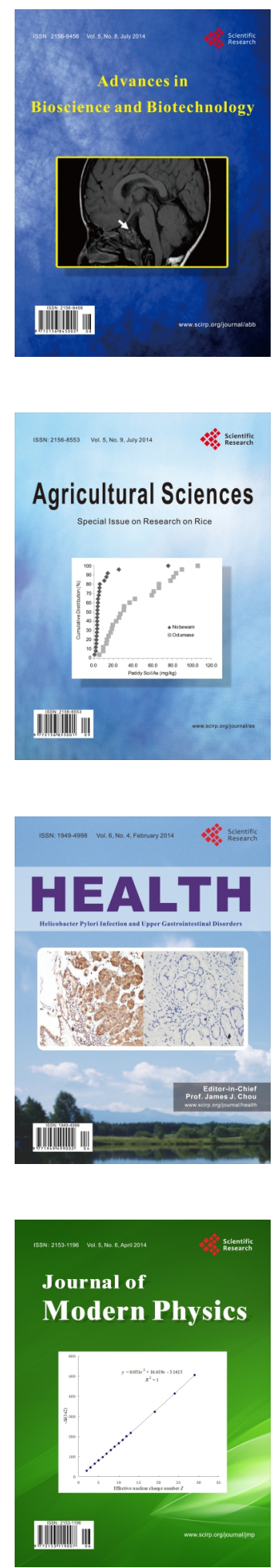
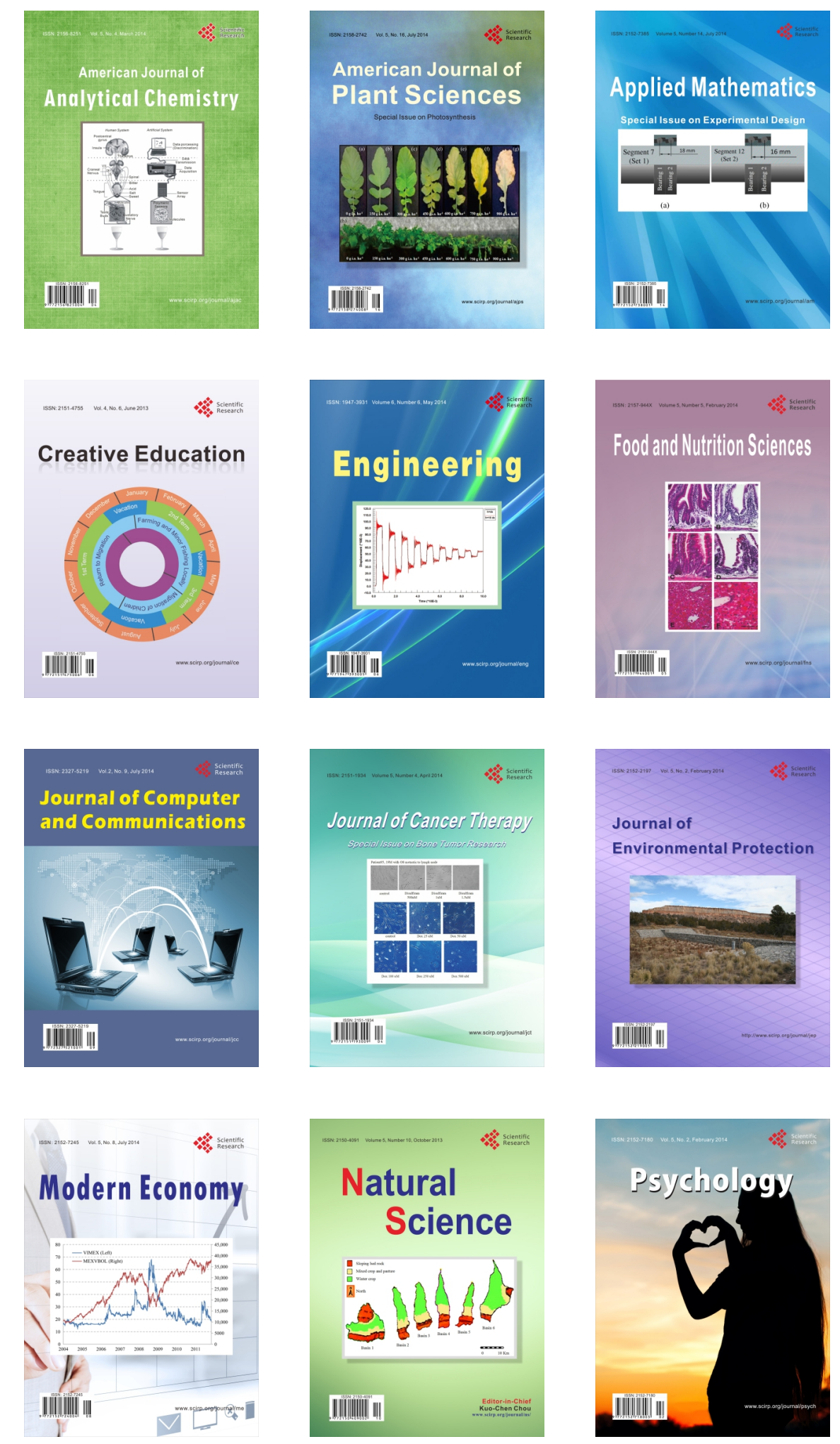